\title{
Investigation of the Shell Effect on Neutron Induced Cross Section of Actinides
}

\author{
Kabiru Muhammed ${ }^{1}$, M. Y. Onimisi ${ }^{1, *}$, S. A. Jonah ${ }^{2}$ \\ ${ }^{1}$ Department of physics, Nigerian Defence Academy, P.M.B 2109 Kaduna, Nigeria \\ ${ }^{2}$ Centre for Energy Research and Training (CERT), Ahmadu Bello University, Zaria, Kaduna, Nigeria
}

\begin{abstract}
Investigations has been made concerning the development of the effect of shell structure on neutron induced cross section, evaluations for Actinides elements in the energy range of 0-30 MeV. The EXIFON code, which is based on the analytical model for statistical multistep direct and multistep compound reactions, was used for the calculation of the cross section for $(n-2 n),(n-p)$ and $(n-\alpha)$ reaction channels. Results are compared with data from the experimental database, EXFOR from the IAEA nuclear data bank to deduce the shell effect. An empirical relation for the reaction cross section has been established for magic numbers nucleus in the (n-2n) reaction channel in the energy range. Results shows that the odd-even effect has also been observed as the cross section for odd-even nuclei are higher than their neighbouring even-even nuclei and with comparison in term of Shell correction, the cross section is higher when the Shell correction is not considered.
\end{abstract}

Keywords Neutron Induce Reaction, Cross Section, Shell Effect, Exifon Code, Exfor

\section{Introduction}

Neutron-induced fission reaction is the most important processes that occur in reactors, accelerator-driven systems and nuclear explosions. In order to develop concepts of these reactions, it is necessary to have clear knowledge and nuclear data of the reaction cross-sections for structural materials, fissile actinides and most important fission products in a given energy range. In a nuclear reactor, $[3,6]$ the main types of elements found during or after a reaction are fission products, actinides, and activation products. Fission products are the largest source of radioactivity for the first several hundred years, while actinides are dominant roughly $10^{3}$ to $10^{5}$ years after fuel use, Even though hazards are associated with radioactivity of actinides, many beneficial applications exist as well such as Radioactive nuclides are used in cancer therapy, analytical chemistry, in basic research in the study of chemical structures and mechanisms. [6] All of the actinide elements are unstable toward radioactive decay; the reason that actinium, thorium, protactinium, and uranium are found in nature at all is because some of their isotopes are unusually stable and others are being formed constantly by decay of the long-lived isotopes.[3]

In this present work, the cross section of Actinides elements was calculated in a neutron energy range from $0-$ $31 \mathrm{MeV}$ to investigate the effect of shell structure on neutron induced cross section using Exifon code.

* Corresponding author:

onimisimy@yahoo.com (M. Y. Onimisi)

Published online at http://journal.sapub.org/jnpp

Copyright (C) 2011 Scientific \& Academic Publishing. All Rights Reserved
The code which is based on an analytical model for statistical multistep direct and multistep compound approach reaction was use for the calculation of the cross section of the group of elements for $(n-2 n),(n-p)$ and $(n-\alpha)$ reaction channels with shell correction as the only adjustable parameter[10,14]. Result were compared between calculation with shell correction and without shell correction and also compared with experimental data, EXFOR from the IAEA nuclear data bank.

\section{Calculation}

The Exifon code is based on the analytical model for statistical multi step direct and multistep compound reaction (SMD/SMC). It predicts emission spectra, angular distributions, and activation cross section including equilibrium, Pre-equilibrium, as well as direct (collective and non- collective) processes. Multiple particle emission is considered up to three decay of the compound system. The model is restricted to neutron, proton, and alpha induced reaction with neutron, proton, alpha, photon in the outgoing channels. The range of validity is with a mass numbers $\mathrm{A}>20$, bombarding energies below $100 \mathrm{MeV}$.[10]

A unique description of $(\mathrm{a}, \mathrm{xb})$ emission spectra where $\mathrm{a}, \mathrm{b}$ $=\mathrm{n}, \mathrm{p}$, alpha and gamma (neutron, proton, alpha, and gamma ray) as well as excitation functions (activation cross section) is proposed within a pure statistical multi step model. This approach is based on the many body theory (Green's function formalism) and random matrix physics.

The Exifon code (version 2.0) is a generalization of the energies up to $100 \mathrm{MeV}$. It is a fast code which predicts cross 
section from global parameters set. The output of Exifon can also be arranged into ENDF-6-format.[10]

The following abbreviation will be used: $\mathrm{E}=\mathrm{Ea}+\mathrm{Ba}$ and $\mathrm{U}=\mathrm{Ea}+\mathrm{Ba}-\mathrm{Bb}-\mathrm{Eb}$. Where the excitation energies of the binding and kinetic energies of the ingoing $(\mathrm{c}=\mathrm{a})$ and outgoing $(\mathrm{c}=\mathrm{b})$ particles.

The calculations were performed by code Exifon for the different isotopes of the Actinides groups. Although not all the Nuclei have data in the code, only the following are considered.

\section{\begin{tabular}{|l|l|l|l|}
\hline Uranium-235 & Uranium-238 & Thorium-232 & Plutonium-239 \\
\hline
\end{tabular}}

Base on the above available data in the Code, the following possible activation cross-section of neutron induced reaction, (n, a), (n, p) and (n, 2n), that is (neutron-alpha, neutron-proton, and neutron-neutron respectively) were considered in the energy range of $0-31 \mathrm{MeV}$.

The EXIFON code installed on a personal computer has time for one full-scale description per nucleus (which includes all activation cross-section plus emission spectra from zero up to $31 \mathrm{MeV}$ incident energy) which strongly depends on the incident energy.[10]

Running the program, the input and output directory must first be defined, then the target nucleus specified. The incident particle (in this case Neutron) selected followed by selecting the excitation function in the general option section for this calculation.

The number of incident energy is then specified followed by the first incident energy (in $\mathrm{MeV}$ ), and then the incident energy step is also specified (in $\mathrm{MeV}$ ).

In the modification section, the shell structure effect which is the most important part of this calculation is selected; in it has two options which are WITH and WITHOUT shell effect.

First, the option with shell effect is selected for each target nucleus, an output data (OUTEXI) for the calculation is then stored in the set output directory. Also DAT file name A2N, ALF, and AP (for Neutron-2Neutron, Neutron-Alpha, and Neutron-Proton reaction respectively) are stored in the set output directory.

Secondly, the option without shell effect is then also for each target nucleus, also an output data (OUTEXI) for the calculation is then stored in the set output directory. Also DAT file name A2N, ALF, and AP (for Neutron-2Neutrons, Neutron-Alpha, and Neutron-Proton reaction respectively) are stored in the set output directory. The Date in the DAT files can then be plotted in the form of chats or graphs or viewed using a DAT file Viewer.

\section{Results/Observation}

Plutonium - 239 which has an Even-Odd Nucleus (proton-94, neutron-145) and it is clearly seen that in the energy range of $0-31 \mathrm{MeV}$, the $(\mathrm{n}-2 \mathrm{n})$ reaction is the most dominant reaction channel in the interaction of neutron with medium and heavy mass isotopes. Looking at the data from the table 1 below, the $(n-2 n)$ reaction has the highest cross section in $11 \mathrm{MeV}$ also with comparison in term of the shell correction, it shows that cross section is higher when the shell correction is not considered at the given energy range.

Table 1. Cross section of PU-239 with energy range of $1 \mathrm{MeV}$ to $31 \mathrm{MeV}$ from EXIFON Code

\begin{tabular}{|c|c|c|c|c|c|c|c|}
\hline \multirow[t]{2}{*}{$\begin{array}{c}\text { Ele } \\
\text { ment }\end{array}$} & \multirow[t]{2}{*}{$\begin{array}{l}\text { Energy } \\
(\mathrm{MeV})\end{array}$} & \multicolumn{3}{|c|}{$\begin{array}{c}\text { Cross Section With Shell } \\
\text { Correction (mb) }\end{array}$} & \multicolumn{3}{|c|}{$\begin{array}{l}\text { Cross Section Without } \\
\text { Shell Correction (mb) }\end{array}$} \\
\hline & & $(n-2 n)$ & (n-p) & $(\mathrm{n}-\boldsymbol{\alpha})$ & $(n-2 n)$ & (n-p) & $(\mathrm{n}-\boldsymbol{\alpha})$ \\
\hline \multirow{16}{*}{$\begin{array}{l}\text { PU- } \\
239\end{array}$} & 1.00 & 0.00 & 0.00 & 0.00 & 0.00 & 0.00 & 0.00 \\
\hline & 3.00 & 0.00 & 0.00 & 0.00 & 0.00 & 0.00 & 0.00 \\
\hline & 5.00 & 0.00 & 0.00 & 0.00 & 0.00 & 0.00 & 0.00 \\
\hline & 7.00 & 231.98 & 0.00 & 0.00 & 266.24 & 0.00 & 0.00 \\
\hline & 9.00 & 1347.80 & 0.00 & 0.00 & 1373.20 & 0.00 & 0.00 \\
\hline & 11.00 & 1633.80 & 0.23 & 0.00 & 1643.50 & 0.23 & 0.00 \\
\hline & 13.00 & 1627.70 & 1.48 & 0.00 & 1625.50 & 1.48 & 0.00 \\
\hline & 15.00 & 510.98 & 4.58 & 0.00 & 506.22 & 4.57 & 0.00 \\
\hline & 17.00 & 94.34 & 9.70 & 0.00 & 96.82 & 9.63 & 0.00 \\
\hline & 19.00 & 25.12 & 15.37 & 0.01 & 26.59 & 15.20 & 0.01 \\
\hline & 21.00 & 10.68 & 20.00 & 0.02 & 11.44 & 19.71 & 0.02 \\
\hline & 23.00 & 6.00 & 23.24 & 0.05 & 6.50 & 22.85 & 0.05 \\
\hline & 25.00 & 3.87 & 25.45 & 0.08 & 4.19 & 24.99 & 0.08 \\
\hline & 27.00 & 2.67 & 26.92 & 0.12 & 2.90 & 26.40 & 0.12 \\
\hline & 29.00 & 1.90 & 25.80 & 0.15 & 2.07 & 25.39 & 0.15 \\
\hline & 31.00 & 0.89 & 0.00 & 0.00 & 1.01 & 0.00 & 0.00 \\
\hline
\end{tabular}

Table 2. Cross section of Th-232 with energy range of $1 \mathrm{MeV}$ to $31 \mathrm{MeV}$ from EXIFON Code

\begin{tabular}{|c|c|c|c|c|c|c|c|}
\hline \multirow[t]{2}{*}{$\begin{array}{c}\text { Ele- } \\
\text { ment }\end{array}$} & \multirow[t]{2}{*}{$\begin{array}{l}\text { Energy } \\
(\mathrm{MeV})\end{array}$} & \multicolumn{3}{|c|}{$\begin{array}{l}\text { Cross SectionWith Shell } \\
\text { Correction (mb) }\end{array}$} & \multicolumn{3}{|c|}{$\begin{array}{l}\text { Cross Section Without } \\
\text { Shell Correction (mb) }\end{array}$} \\
\hline & & $(n-2 n)$ & $(\mathrm{n}-\mathrm{p})$ & $(\mathrm{n}-\boldsymbol{\alpha})$ & $(n-2 n)$ & $(n-p)$ & $(\mathrm{n}-\boldsymbol{\alpha})$ \\
\hline \multirow{16}{*}{$\begin{array}{l}\text { Th- } \\
232\end{array}$} & 1.00 & 0.00 & 0.00 & 0.00 & 0.00 & 0.00 & 0.00 \\
\hline & 3.00 & 0.00 & 0.00 & 0.00 & 0.00 & 0.00 & 0.00 \\
\hline & 5.00 & 0.00 & 0.00 & 0.00 & 0.00 & 0.00 & 0.00 \\
\hline & 7.00 & 1.08 & 0.00 & 0.00 & 1.60 & 0.00 & 0.00 \\
\hline & 9.00 & 786.15 & 0.00 & 0.00 & 851.29 & 0.00 & 0.00 \\
\hline & 11.00 & 1695.80 & 0.00 & 0.00 & 1711.40 & 0.00 & 0.00 \\
\hline & 13.00 & 1896.40 & 0.08 & 0.00 & 1901.10 & 0.08 & 0.00 \\
\hline & 15.00 & 826.35 & 0.72 & 0.00 & 724.18 & 0.72 & 0.00 \\
\hline & 17.00 & 138.24 & 2.74 & 0.00 & 119.57 & 2.74 & 0.00 \\
\hline & 19.00 & 30.92 & 6.45 & 0.02 & 27.80 & 6.38 & 0.02 \\
\hline & 21.00 & 11.88 & 11.35 & 0.05 & 11.06 & 11.11 & 0.06 \\
\hline & 23.00 & 6.52 & 16.03 & 0.10 & 6.17 & 15.53 & 0.11 \\
\hline & 25.00 & 4.25 & 19.82 & 0.17 & 4.04 & 19.26 & 0.18 \\
\hline & 27.00 & 2.98 & 22.43 & 0.26 & 2.85 & 21.73 & 0.27 \\
\hline & 29.00 & 2.19 & 24.29 & 0.35 & 2.09 & 23.48 & 0.36 \\
\hline & 31.00 & 1.64 & 25.59 & 0.43 & 1.58 & 24.70 & 0.44 \\
\hline
\end{tabular}

Thorium - 232 which has an Even-Even Nucleus (proton-90, neutron-142), it is clearly seen that in the energy range of $0-31 \mathrm{MeV}$, the $(\mathrm{n}-2 \mathrm{n})$ reaction is the most dominant reaction channel in the interaction of neutron with medium and heavy mass isotopes. Looking at the data from the table 2 above, the $(n-2 n)$ reaction has the highest cross section in $13 \mathrm{MeV}$ and with comparison in term of the shell correction, it shows that cross section is higher when the shell correction is not considered at the given energy range.

Uranium - 235 which has an Even-Odd Nucleus (proton-92, neutron-143) and it is clearly seen that in the energy range of $0-31 \mathrm{MeV}$, the $(\mathrm{n}-2 \mathrm{n})$ reaction is the most dominant reaction channel in the interaction of neutron with medium 
and heavy mass isotopes. Looking at the data from the table 3 below, the $(n-2 n)$ reaction has the highest cross section in $11 \mathrm{MeV}$. With comparison in term of the shell correction, it shows that cross section is higher when the shell correction is not considered at the given energy range.

Uranium - 238 which has an Even-Even Nucleus (proton-92, neutron-146) with a neutron magic number, it is clearly seen that in the energy range, there is only one possible reaction (n-p) and the shell correction has no effect because of the highly stability of the nucleus.

Table 3. Cross section of U-232 with energy range of $1 \mathrm{MeV}$ to $31 \mathrm{MeV}$ from EXIFON Code

\begin{tabular}{|c|c|c|c|c|c|c|c|}
\hline \multirow[t]{2}{*}{ Element } & \multirow[t]{2}{*}{$\begin{array}{r}\text { Energy } \\
(\mathrm{MeV})\end{array}$} & \multicolumn{3}{|c|}{$\begin{array}{c}\text { Cross Section With Shell } \\
\text { Correction (mb) }\end{array}$} & \multicolumn{3}{|c|}{$\begin{array}{l}\text { Cross Section Without } \\
\text { Shell Correction (mb) }\end{array}$} \\
\hline & & $(n-2 n)$ & $(n-p)$ & $(\mathrm{n}-\boldsymbol{\alpha})$ & $(n-2 n)$ & (n-p) & $(\mathbf{n}-\boldsymbol{\alpha})$ \\
\hline \multirow{16}{*}{ U-235 } & 1.00 & 0.00 & 0.00 & 0.00 & 0.00 & 0.00 & 0.00 \\
\hline & 3.00 & 0.00 & 0.00 & 0.00 & 0.00 & 0.00 & 0.00 \\
\hline & 5.00 & 0.00 & 0.00 & 0.00 & 0.00 & 0.00 & 0.00 \\
\hline & 7.00 & 710.46 & 0.00 & 0.00 & 777.96 & 0.00 & 0.00 \\
\hline & 9.00 & 1675.60 & 0.00 & 0.00 & 1693.30 & 0.00 & 0.00 \\
\hline & 11.00 & 1829.40 & 0.10 & 0.00 & 1838.60 & 0.10 & 0.00 \\
\hline & 13.00 & 1436.70 & 0.91 & 0.00 & 1397.70 & 0.91 & 0.00 \\
\hline & 15.00 & 285.84 & 3.34 & 0.00 & 281.02 & 3.33 & 0.00 \\
\hline & 17.00 & 52.62 & 7.58 & 0.00 & 54.55 & 7.53 & 0.00 \\
\hline & 19.00 & 16.31 & 12.58 & 0.01 & 17.48 & 12.44 & 0.01 \\
\hline & 21.00 & 7.87 & 16.88 & 0.03 & 8.56 & 16.62 & 0.03 \\
\hline & 23.00 & 4.76 & 19.94 & 0.07 & 5.23 & 19.57 & 0.07 \\
\hline & 25.00 & 3.22 & 22.05 & 0.11 & 3.54 & 21.60 & 0.11 \\
\hline & 27.00 & 2.28 & 23.47 & 0.16 & 2.51 & 22.96 & 0.16 \\
\hline & 29.00 & 1.66 & 24.40 & 0.21 & 1.83 & 23.84 & 0.21 \\
\hline & 31.00 & 0.96 & 7.10 & 0.24 & 1.10 & 7.75 & 0.24 \\
\hline
\end{tabular}

Table 4. Cross section of Th-232 with energy range of $1 \mathrm{MeV}$ to $31 \mathrm{MeV}$ from EXIFON Code

\begin{tabular}{|c|c|c|c|c|c|c|c|}
\hline Element & $\begin{array}{c}\text { Energy } \\
(\mathbf{M e V})\end{array}$ & \multicolumn{2}{|c|}{$\begin{array}{c}\text { Cross Section With Shell } \\
\text { Correction (mb) }\end{array}$} & \multicolumn{3}{c|}{$\begin{array}{c}\text { Cross Section Without } \\
\text { Shell Correction (mb) }\end{array}$} \\
\hline \multirow{4}{*}{} & & $\mathbf{( n - 2 n )}$ & $\mathbf{( n - p )}$ & $\mathbf{( n - \alpha )}$ & $\mathbf{( n - 2 n )}$ & $\mathbf{( n - p )}$ & $\mathbf{( n - \alpha )}$ \\
\hline \multirow{4}{*}{$\begin{array}{c}\text { U- } \\
238\end{array}$} & 1.00 & 0.00 & 0.00 & 0.00 & 0.00 & 0.00 & 0.00 \\
\cline { 2 - 8 } & 3.00 & 0.00 & 0.00 & 0.00 & 0.00 & 0.00 & 0.00 \\
\cline { 2 - 8 } & 5.00 & 0.00 & 0.00 & 0.00 & 0.00 & 0.00 & 0.00 \\
\cline { 2 - 8 } & 7.00 & 0.00 & 0.00 & 0.00 & 0.00 & 0.00 & 0.00 \\
\cline { 2 - 8 } & 9.00 & 0.00 & 0.00 & 0.00 & 0.00 & 0.00 & 0.00 \\
\cline { 2 - 8 } & 11.00 & 0.00 & 0.00 & 0.00 & 0.00 & 0.00 & 0.00 \\
\cline { 2 - 8 } & 13.00 & 0.00 & 0.00 & 0.00 & 0.00 & 0.00 & 0.00 \\
\cline { 2 - 8 } & 15.00 & 0.00 & 0.50 & 0.00 & 0.00 & 0.50 & 0.00 \\
\cline { 2 - 8 } & 17.00 & 0.00 & 2.90 & 0.00 & 0.00 & 2.90 & 0.00 \\
\cline { 2 - 8 } & 19.00 & 0.00 & 9.60 & 0.00 & 0.00 & 9.60 & 0.00 \\
\cline { 2 - 8 } & 21.00 & 0.00 & 22.70 & 0.00 & 0.00 & 22.70 & 0.00 \\
\cline { 2 - 8 } & 23.00 & 0.00 & 43.00 & 0.00 & 0.00 & 43.00 & 0.00 \\
\cline { 2 - 8 } & 25.00 & 0.00 & 69.50 & 0.00 & 0.00 & 69.50 & 0.00 \\
\cline { 2 - 8 } & 27.00 & 0.00 & 100.50 & 0.00 & 0.00 & 100.50 & 0.00 \\
\cline { 2 - 7 } & 29.00 & 0.00 & 134.20 & 0.00 & 0.00 & 134.20 & 0.00 \\
\cline { 2 - 7 } & 31.00 & 0.00 & 169.40 & 0.00 & 0.00 & 169.40 & 0.00 \\
\hline
\end{tabular}

\section{Result Discursions}

From the above experimental data for the (n-2n), (n-p) and $(n-\alpha)$ reactions cross section in the energy range of $0-31$ $\mathrm{MeV}$, it is generally observed that The dominant reaction channel in the interaction of neutron with heavy isotopes is $(n-2 n)$ reaction channel with the exception of Uranium-238 (which has a highly stable Nucleus), in the energy range there is only a slit probability of proton been emitted).
Analysis of result from Tables $1-4$ shows that, Generally, the even-odd nuclei across the group have higher cross section than their neighbouring even-even nuclei that is the higher the mass number the less the probability of the reaction occurring, therefore the cross section decreases with increases in mass number and with comparison in term of Shell correction, the cross section is higher when the Shell correction is not considered.

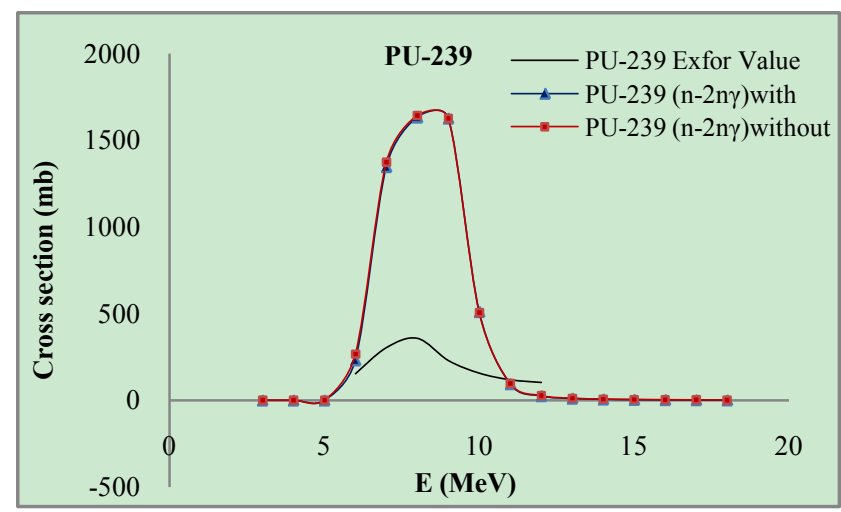

Figure 1. Graphical representation of the comparison between the Exofor and the Calculated values for $(2,2 \mathrm{n})$ reaction channel for PU-239

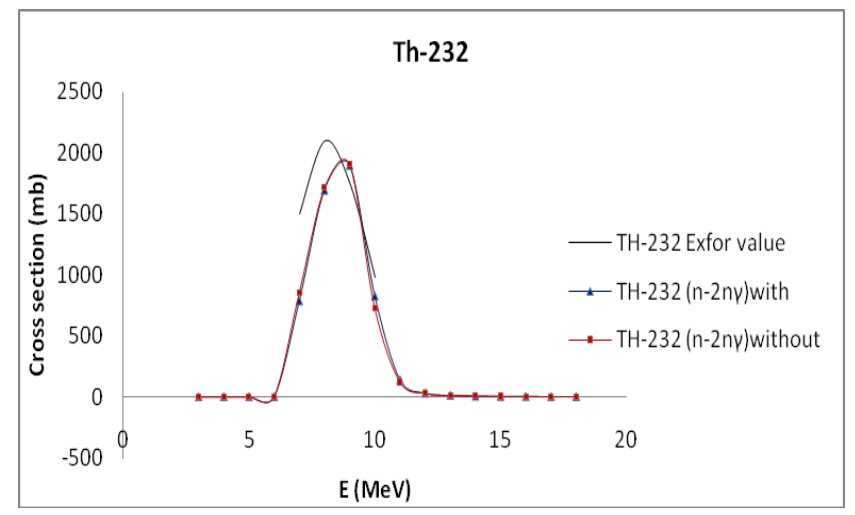

Figure 2. Graphical representation of the comparison between the Exofor and the Calculated values for $(2,2 \mathrm{n})$ reaction channel for Th-232

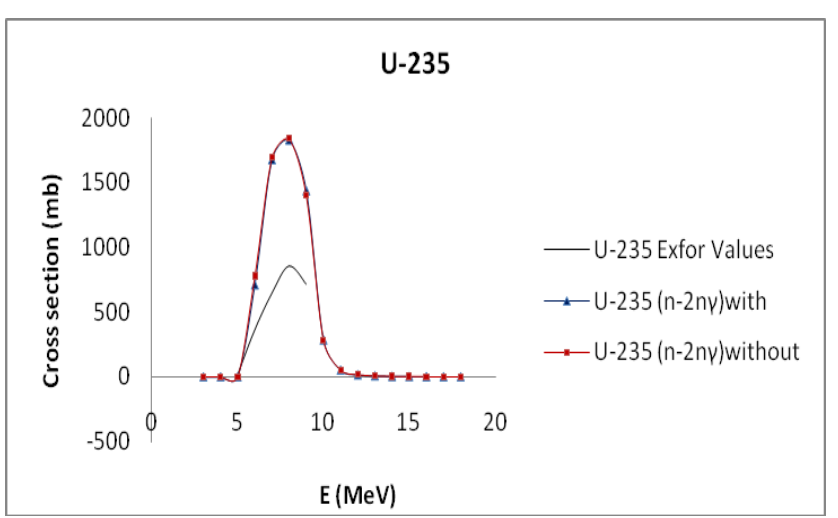

Figure 3. Graphical representation of the comparison between the Exofor and the Calculated values for $(2,2 \mathrm{n})$ reaction channel for Th-232

A comparison of experimental data from EXFOR data Library and the calculated result for $(n-2 n)$ reaction channel (Fig 1-3) it is clearly seen that, generally the reaction deviate from the experimental data with the exception of U-235 which have similar pathway in both consideration (Fig 3). 
Also the values where the shell correction is considered tend to move closer to the experimental values.

\section{REFERENCES}

[1] A. Iwamoto and K. Harada. Proceed. Of the international conference on nuclear data for science and technology, Mito Japan. Page 485, 1988

[2] A. V. Ignatyuk, V. P. Lunev, Yu. N. Shubin, E. V. Gai and N. N. Titarenko "Neutron Cross section evaluation for ${ }^{238}$ U up to $150 \mathrm{MeV}$ " Institute of physics and power engineering, Russia (2000)

[3] Actinide element. (2009). In Encyclopædia Britannica. Retrieved June 02, 2009,from Encyclopædia Britannica Online: http://www.britannica.com/EBchecked/topic/4354/actinide-e lement

[4] Bormann M, Neuert H \& Scobel W, IAEA Handbook on nuclear activation cross sections, Technical Report No. 156, Vienna, Austria, (1974), p.87

[5] Cullen D E, Howerton R J, Perkins S T \& Mac Gnegor M H, Major neutron induced interaction cross sections, Report No.UCRL-50400,7 and 8(Lawrence Livermore National Laboratory, Univ. Of California, Barkley)

[6] From Wikipedia,(2009) the freeencyclopedia http://en.wikipedia.org/wiki/Actinoid\#cite_note-0

[7] H. Kalka, M. Torjman, H.N Lien, R. Lopez, and D. Seeliger. Description of (n-p) and (n-2n) activation cross sections for medium mass nuclei within statistical multistep theory. Sektion Physik, Tech University, Germany. 1989

[8] Hauser. W. And Feshbach. H. 1952: Inelastic scattering of neutrons phys. Rev. $87,366-373$

[9] Iskender A. R, Measurement and Statistical model calculations of activation cross section for $\left.{ }^{19} \mathrm{~F}(n, p)\right)^{19} \mathrm{O}$ reaction between 13.6 and $14.9 \mathrm{MeV}$ neutron energies. Cekmece nuclear research and training centre, Turkey (2003)

[10] Kalka, H. 1991: EXIFON - A Statistical Multi-step reaction code, Report, Technische University Dresden, Germany (1991)

[11] L.F Hansen B.A Pohl, C. Wong, Ch. Lagrange. Measurement and calculation of neutron scattering in Actinides Region. Phys.Rev. C34, 2075. 1986

[12] M.G. Mayer and J.H.D. Jensen, Elementary Theory of Nuclear Shell Structure. Wiley, New York, (1955)

[13] P. Ring and P. Schuck. The nuclear many body problems. Springer Verlag, New York, 1980

[14] P.G Young, E.D Arthur M.B Chadwick, Nuclear model calculation theory and the use of codes. IAEA workshop on Nuclear reaction data and nuclear reactors. Trieste Italy. 1998 Vol 1

[15] S.L Goyal and P. Gur. Empirical relation and establishment of shell effect in $(\mathrm{n}-2 \mathrm{n})$ reaction cross section at $14 \mathrm{MeV}$. Guru Jambheshwar University of Science and Tech. India, 2008

[16] T.A Brody, J. Flores, J.B French, P.A Mello, A. Pandey, and S.M Wong. Rev. Mod. Phys. 53.385, 1981

[17] Talou, P., Kawano, T., Young, P. G., Chadwick, M. B., and MacFarlane, R. E., Improved Evaluations of Neutron Induced Reactions on Americium Isotopes. Nucl. Sci. Eng. (2006, in print). Los Alamos report LA-UR-06-0316 (2006)

[18] V.A. Konshin. Calculation of neutron and proton induced reaction cross section for Actinides in the energy region from $10 \mathrm{Mev}$ to $1 \mathrm{GeV}$. Report JAERI-Research- 95-036, Japan Atomic energy research institute. 1995 